\title{
Stress Simulation Analysis and Study of Glass Capillary with Displacement Load
}

\author{
Bi-cheng Wu ${ }^{1}$, Zhi-yuan Yao, ${ }^{2}$ \\ ${ }^{1}$ State Key Laboratory of Mechanics and Control of Mechanical Structures, Nanjing University of \\ Aeronautics and Astronautics,29 Yudao Street, Nanjing, China \\ ${ }^{2}$ State Key Laboratory of Mechanics and Control of Mechanical Structures, Nanjing University of \\ Aeronautics and Astronautics,29 Yudao Street, Nanjing, China \\ ${ }^{*}$ Corresponding author
}

Keywords: Simulation, Stress, Capillary, Load.

\begin{abstract}
In order to solve the problem that the tip of the capillary after stretch is blocked, we need to deal with the tip to make the fracture occurs on the tip. A model is established to analyze the Mises stress in the capillary under the displacement load. Two bodies are assembled to apply displacement load on both sides of the capillary. The left surface of the capillary is fixed. The biggest Mises stress values of units in the process of applying displacement load can be figured out which are all more than $1.08 \times 10^{10} \mathrm{~Pa}$. The Mises stress values are greater to make the tip of the capillary fractured. The simulation analysis and study provide the theoretical feasibility for the manufacture of micro-nano channels.
\end{abstract}

\section{Introduction}

The base of the third generation DNA sequencing is the pore fluid device of the nanopores[1-5]. For the time being, there are two kinds of nanopores for the DNA sequencing. One is the biological nanopore, and the other is the solid-state nanopore. The preparation of solid-state nanopores are mainly the following several ways. One is the use of high energy electron beam or focused beam, drilling in the specified location of nano film materials directly out of the nanopores. So we can get an ideal nanopore. But the drawbacks of this approach are its high cost and the complicated experimental equipment. As a result, this way can only be suitable for the laboratory research and cannot be applied in business widely.

Stretching the glass tube after heating[6-8]is a new kind of method for the preparation of nanopores. The capillary can be drawn out by the capillary drawing system which is based on laser heating. And the inner diameter of the tip of the capillary is under $1 \mu \mathrm{m}$. But it shows that the tip is usually blocked under a microscope observation.

In order to manufacture the micro-nano channels, we need to deal with the tip of the capillary to make fracture occurs in the tip. So that the blocked tip of the capillary can be removed. In this case, the stress of the capillary is the mechanical stress. And the glass is almost an ideal elastic material. So we can describe the mechanical behavior of glass with the theory of linear elastic fracture mechanics. Therefore, this paper proposes an analysis method of applying displacement load on the both sides of the glass elastic rod, aiming at analyzing the stress distribution of the rod. The relative distance of the applied forces has a great influence on the tendency of the mechanical stress and size for the capillary.

\section{Establishment of the Model}

The size of the capillary is in the micron scale. The sample of a capillary which is drawn out by the capillary glass tube drawing system is shown in Fig.1. The inner diameter of the sample is measured in Fig.1, which is $0.48 \mu \mathrm{m}$. 


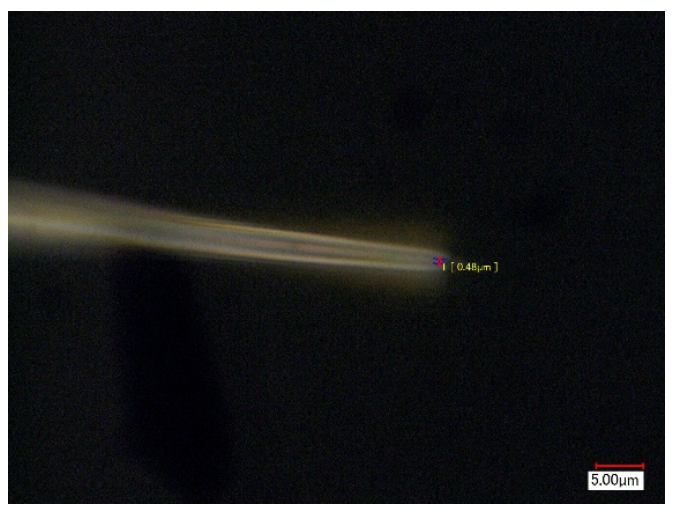

Figure 1. the size of the capillary

So we should refer to the size. The Solid Works model of the capillary is set up. Its length is $0.2 \mathrm{~mm}$ and the outer diameter is $0.002 \mathrm{~mm}$. The material is glass. The Solid Works model for the body which will apply pressure on both sides of the capillary is established. The size of the upper rectangle of the body is $0.004 \times 0.002 \mathrm{~mm}$, the bottom is a semicircle which will apply the displacement load to the capillary. And the material is steel. The parameters and properties of two kinds of materials are shown in Table 1.

Table 1. The parameters and properties

\begin{tabular}{ccccc}
\hline Part & Material & Density $/ \mathrm{kg} \cdot \mathrm{m}^{-3}$ & $\begin{array}{c}\text { Modulus of } \\
\text { elasticity } / \mathrm{GPa}\end{array}$ & $\begin{array}{c}\text { Poisson's } \\
\text { ratio }\end{array}$ \\
\hline Body & No.45 steel & 7800 & 200 & 0.3 \\
Capillary & glass & 2500 & 55 & 0.25 \\
\hline
\end{tabular}

After applying grids on the bodies and the capillary, the grid faults should be checked and avoided. The meshed parts are shown in Fig.2 and Fig.3.

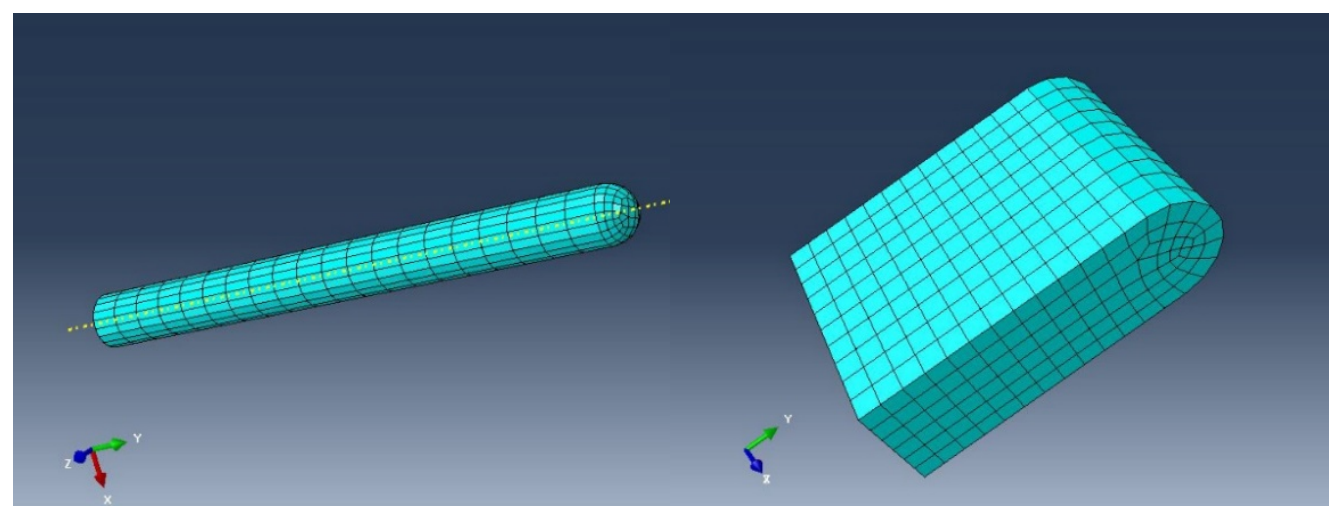

Figure 2. The capillary

Figure 3. The body

The capillary and the bodies are assembled, the general assembly drawing are shown in Fig.4 and Fig.5.

The left surface of the capillary is a fixed surface and the right side is a free hemispherical surface. The two bodies are arranged on both sides of the capillary as are shown in Fig.5. The distance between the lower body and the left surface of the capillary is D which is a fixed value. $\mathrm{D}=0.016 \mathrm{~mm}$. The drepresents the horizontal distance between the two bodies and it is adjustable.

After assembly, the $d$ is set to be $0.006 \mathrm{~mm}$. The stress distribution on the bottom of the glass tube is studied. Initially, a certain distance occurs between the capillary and the lower body. The contact between the capillary and the upper body is frictional. The coefficient of friction $\mu$ is 0.5 . Then, when the lower body moves upwards, Frictional contact will occur between the lower body and the capillary. 


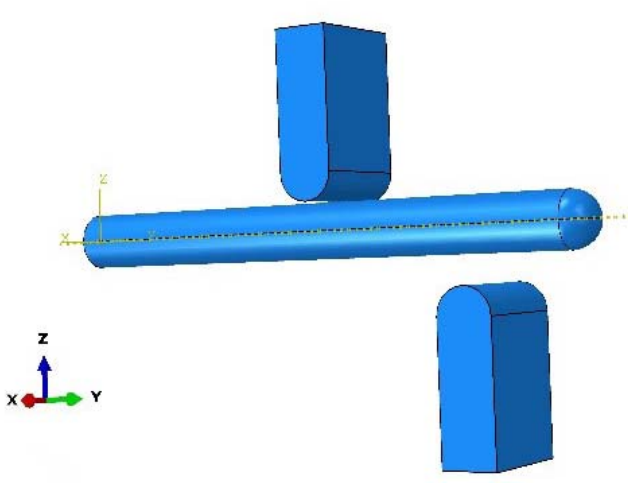

Figure 4.The three dimensional figure

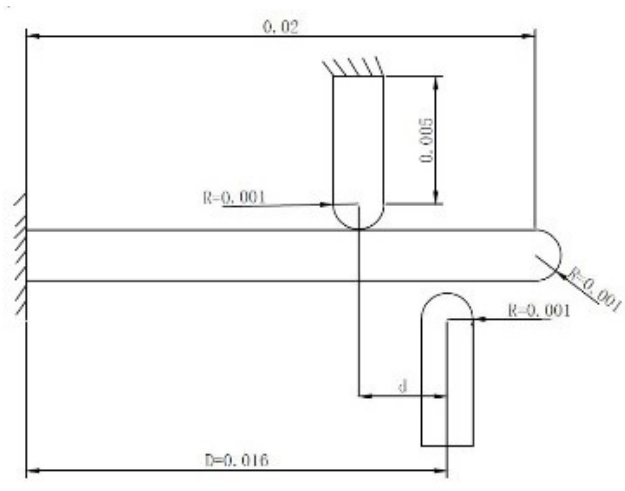

Figure5. The main view

\section{Imposing Constraints and Results Analysis}

The six degrees of freedom of the left surface of the capillary are restrained. In the same way, the six degrees of the upper rectangle of the upper body are restrained. The degrees of freedom of the semicircle surface of the lower body is defined as follows.U3 $=0.01 \mathrm{~mm}, \mathrm{U} 1=\mathrm{U} 2=0$, $\mathrm{UR} 1=\mathrm{UR} 2=\mathrm{UR} 3=0$. In these constraints, the lower body can only move along the $\mathrm{Z}$ axis direction.

A new task is created and submitted. And the results are outputted.The stress cloud pictures are shown in Fig.6and Fig 7.

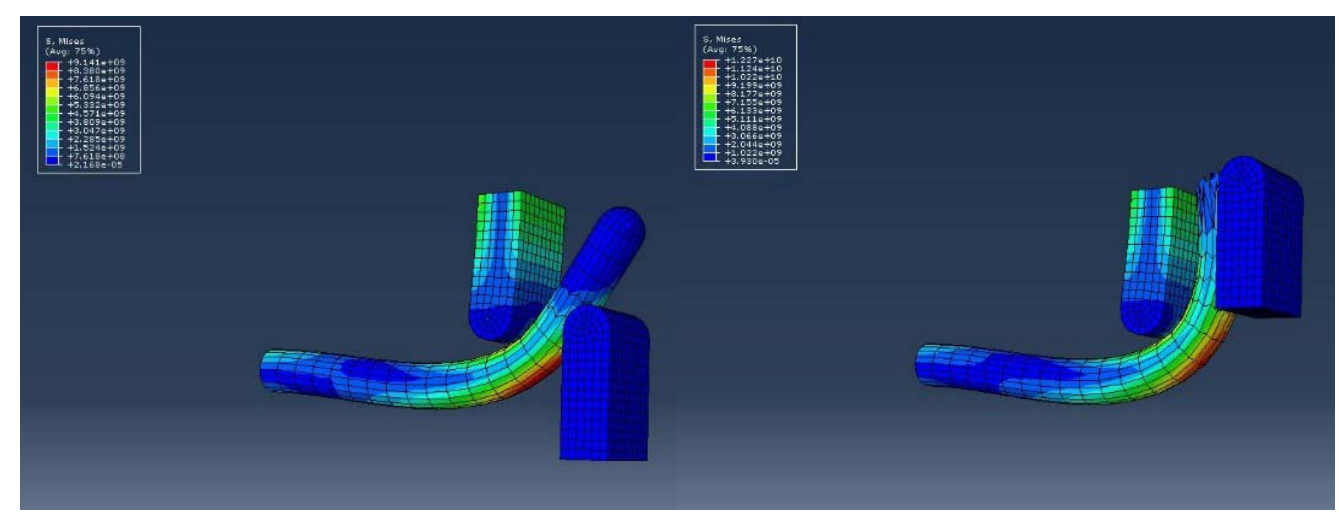

Figure 6. The stress state of intermediate process

Figure 7. The final stress state

In the ABAQUS analysis, the displacement load of U3 is applied in 35 small steps. The 8 units on the bottom of the capillary are selected. their number are574,494,414,334,618,538,458,378, as are shown in Fig.8.

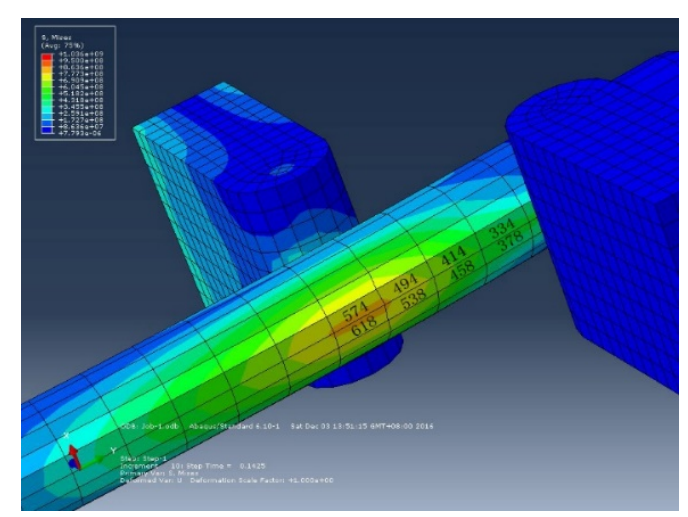

Figure 8. Selected units

The biggest Mises stress values in the process of applying displacement load can be figured out. 
They are all more than $1.08 \times 10^{10} \mathrm{~Pa}$. The theoretical strength of glass can be figured out in different ways which is mainly $10^{10} \sim 1.5 \times 10^{10} \mathrm{~Pa}$. Because of the complexity in the structure of the crystalline and amorphous material, the theory of material strength can be figured out according $\sigma_{\mathrm{th}}=x \cdot E$ approximately. The $\mathrm{E}$ is the modulus of elasticity. The $x$ is a constant related to the material structure. Usually $x=0.1 \sim 0.2$. According to this equation, the theoretical strength of glass is mainly $5.5 \times 10^{9} \mathrm{~Pa}$.

\section{Conclusion}

Comparing the biggest Mises stress values and the theoretical strength of glass, we can get the conclusion that the displacement load can cause the mechanical stress in the capillary and the Mises stress values in the bottom of the capillary are greater to make the capillary fractured. The simulation analysis and study provide the theoretical feasibility for the manufacture of micro-nano channel.

\section{Acknowledgements}

The work was financially supported by NSFC (Grant No.51275229) and National Major Scientific Instruments and Equipment Development Projects (Grant No.2012YQ100225).

\section{References}

[1] Takmakov P, Vlassiouk I, Smirnov S. Hydrothermally shrunk alumina nanopores and their application to DNA sensing.[J]. Analyst, 2006, 131(11):1248-53.

[2] Khulbe P K, Mansuripur M, Gruener R. DNA translocation through $\alpha$-hemolysin nanopores with potential application to macromolecular data storage[J]. Journal of Applied Physics, 2005, 97(10):104317-104317-7.

[3] Lin X. Preparation and Application of Nanopores for Single Molecule Detection[J]. Progress in Chemistry, 2011, 23(4):800-809.

[4] Benítez-Páez A, Portune K J, Sanz Y. Species-level resolution of 16S rRNA gene amplicons sequenced through the MinIONTM portable nanopore sequencer[J]. GigaScience, 2016, 5(1):1-9.

[5] Li J, Yu D, Zhao Q. Solid-state nanopore-based DNA single molecule detection and sequencing[J]. Microchimica Acta, 2016, 183(3):941-953.

[6] Wei S I, Sha J, Liu L, et al. Detection of $\lambda$-DNA through silicon nitride nanopores[J]. 2014.

[7] Xian-pu Jia.A Research on Driver Control and Test of Extrude System for Nanopore Based on Glass [D].Nanjing University of Aeronautics and Astronautics,2015(in Chinese)

[8] Shan chen. The Design and Experimental Research of the Glass Capillary Tension Feeding System Based on Laser Heating[D]. Nanjing University of Aeronautics and Astronautics,2014(in Chinese) 